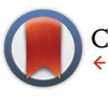

CrossMark

\&lick for updates

Cite this: Food Funct., 2016, 7, 2712

\title{
Food-grade Pickering emulsions stabilised with solid lipid particles
}

\author{
Aleksandra Pawlik, Daniel Kurukji, * Ian Norton and Fotis Spyropoulos
}

Aqueous dispersions of tripalmitin particles (with a minimum size of $130 \mathrm{~nm}$ ) were produced, via a hot sonication method, with and without the addition of food-grade emulsifiers. Depending on their relative size and chemistry, the emulsifiers altered the properties of the fat particles (e.g. crystal form, dispersion state and surface properties) by two proposed mechanisms. Firstly, emulsifiers modify the rate and/or extent of polymorphic transitions, resulting in the formation of fat crystals with a range of polarities. Secondly, the adsorption of emulsifiers at the particle interface modifies crystal surface properties. Such emulsifier-modified fat particles were then used to stabilise emulsions. As the behaviour of these particles was predisposed by the kind of emulsifier employed for their manufacture, the resulting particles showed different preferences to which of the emulsion phases (oil or water) became the continuous one. The polarity of the fat particles decreased as follows: Whey Protein Isolate $>$ Soy Lecithin $>$ Soy Lecithin + Tween 20 > Tween 20 > Polyglycerol Polyricinoleate > no emulsifier. Consequently, particles stabilised with WPI formed oil-in-water emulsions (O/W); particles stabilised solely with lecithin produced a highly unstable W/O emulsion; and particles stabilised with a mixture of lecithin and Tween 20 gave a stable W/O emulsion with drop size up to $30 \mu \mathrm{m}$. Coalescence stable, oil-continuous emulsions (W/O) with drop sizes between 5 and $15 \mu \mathrm{m}$ were produced when the tripalmitin particles were stabilised with solely with Tween 20, solely with polyglycerol polyricinoleate, or with no emulsifier at all. It is proposed that the stability of the latter three emulsions was additionally enhanced by sintering of fat particles at the oilwater interface, providing a mechanical barrier against coalescence.

Received 23rd February 2016, Accepted 7th May 2016

DOI: $10.1039 / c 6 f o 00238 b$

www.rsc.org/foodfunction oil-water interface. ${ }^{1}$ The contact angle is also linked to surfactant adsorption onto the particle's surface. ${ }^{12}$ Ionic surfactants adsorb at the surface of silica particles, induce their flocculation at the water-oil interface, and enhance emulsion stability. ${ }^{13,14}$ Surfactants can also displace particles from an interface, ${ }^{3}$ sometimes without destabilising the emulsion. ${ }^{15}$ In a system where both solid particles (hydrophilic silica) and a low molecular weight surfactant (monoolein) were present, Pichot et al., proposed a two-fold emulsion stabilisation mechanism. ${ }^{6}$ During emulsification a highly dynamic monoolein rapidly reduced interfacial tension, facilitating further droplet break-up and limiting coalescence. This allowed sufficient time for the less mobile silica particles to gather at the oilwater interface, displace monoolein molecules and thus provide long-term emulsion stability against coalescence. Murray et al. demonstrated a synergistic effect between a protein and starch at a liquid-liquid interface. ${ }^{2}$ Without indication of specific protein-protein interactions, it was proposed that a protein could enhance solid particle assembly at the adsorbed layer, and their subsequent jamming at the interface. The resultant increase in interfacial viscoelasticity correlated with an increase in emulsion stability against coalescence and/or Ostwald ripening. Using monoglycerides Frasch-Melnik
School of Chemical Engineering, University of Birmingham, Birmingham, B15 2TT, UK. E-mail: d.j.kurukji@gmail.co.uk; Tel: +44 (0)7414 861610 
et al. $(2010)^{5}$ altered the polarity of inherently hydrophobic triglyceride crystals ${ }^{16}$ and seeded their formation at the interface $^{17}$ during the manufacture of the water-in-oil emulsions. By precisely selecting the ratio of a surface-active crystalline monoglyceride to a network-forming triglyceride as well as the processing conditions (cooling and shearing rates), a sintered "shell" of fat crystals at an interface surrounded by a network of fat crystals in the bulk continuous phase was formed. The interplay of the two mechanisms of fat crystal stabilisation: surface-active (Pickering stabilisation), surface-inactive (network stabilisation), or a combination of both, on the sedimentation and coalescence stability of emulsions, was investigated by Ghosh et al. ${ }^{18}$ They proposed that Pickering crystals were more effective than the network crystals for emulsion stabilisation.

Other surfactants (e.g. polyglycerol polyricinoleate (PGPR) and lecithin) have also been employed to increase aqueous wetting of triglycerides. ${ }^{16,19,20}$ The general approach was to either pre-crystallise the fat within the oil phase followed by emulsion formation or to crystallise the fat post emulsification. In the flash-cooling process Garti et al. ${ }^{20}$ produced the $\alpha$ polymorph of tristearin crystals in a liquid oil, which were more hydrophilic than the $\beta$ polymorph, thus more easily drawn to the interface. ${ }^{16}$ Nevertheless, production of non-flocculated, submicron $\alpha$ fat crystals required addition of a suitable surfactant (PGPR).

In this work, solid lipid particles with tailored properties were manufactured first and then assessed for use as emulsion stabilisers. The aim was to produce aqueous dispersions of fat particles (via a hot emulsification step) designed to possess the required size, morphology, and surface characteristics necessary to function as Pickering stabilisers. To achieve this, food-grade emulsifiers with a range of chemistries (protein, phospholipid, low and high HLB surfactants, and their mixtures) were utilised and investigated for subsequent potential to: (i) stabilise fat particles in an aqueous dispersion and (ii) alter the microstructure and thus the tendency for the particles to form water- or oil-continuous emulsions.

\section{Experimental}

\section{Materials}

Tripalmitin (with purity $\geq 85 \%$ ) and Polyoxyethylene(20)sorbitanmonolaurate (Tween 20) $(\mathrm{HLB}=16.7)$ were purchased from Sigma Aldrich (UK). Whey Protein Isolate (WPI) was kindly donated by DAVISCO Foods International, Inc. (Switzerland) and Lipoid S45 was kindly provided by Lipoid $\mathrm{GmbH}$ (Germany). The composition of Lipoid S45 was reported from manufacturer as $45-50 \%$ soybean phosphatidylcholine and 10-18\% phosphatidylethanolamine; the typical FA composition is $58-65 \%$ linoleic acid, $12-17 \%$ palmitic acid, $8-12 \%$ oleic acid and other fatty acids. Polyglycerol polyricinoleate (PGPR, HLB $=1.5 \pm 0.5$ ) was obtained from Kerry Bioscience (UK) and sunflower oil was purchased from the local supermarket. Distilled water $\left(\mathrm{pH}=6.8,1.63 \mu \mathrm{S} \mathrm{cm}{ }^{-1}\right)$ was used for the experiments. All materials were used without further purification or modification, and all samples were formulated and reported on a weight-by-weight basis $(\mathrm{g} / \mathrm{g})$.

\section{Methods}

Particle preparation. 5\% tripalmitin was added to water with or without $5 \%$ emulsifier (Tween 20, PGPR, WPI, lecithin or their combinations) and heated up to $75{ }^{\circ} \mathrm{C}$, while mixed using a magnetic stirrer. Then, the premix was process with ultrasound using a sonicating probe (Vibra Cell VCX 500 from Sonics, USA) for $3 \mathrm{~min}$ using a $12 \mathrm{~mm}$ probe $(20 \mathrm{kHz}, 95 \%$ amplitude). The dispersion was then cooled down (to $1-3{ }^{\circ} \mathrm{C}$ ) in an ice bath and stored in the fridge $\left(4 \pm 2^{\circ} \mathrm{C}\right)$. Fresh dispersions were allowed to equilibrate for $24 \mathrm{~h}$ before their characterisation was performed.

Emulsion preparation. Oil-in-water emulsions were prepared by adding $20 \mathrm{~g}$ sunflower oil into $80 \mathrm{~g}$ of the tripalmitin particle dispersion. Conversely, water-in-oil emulsions were prepared by adding $20 \mathrm{~g}$ of the particle dispersion into $80 \mathrm{~g}$ of sunflower oil. Such mixtures were then homogenised with a high-shear mixer (Silverson L5M from Silverson Machines, Ltd, UK) for $2 \mathrm{~min}$ at $10000 \mathrm{rpm}$. To avoid shear-induced heating of the sample and melting of the fat particles, the mixture was cooled in an ice bath, while emulsified. The emulsions were analysed directly after preparation and then stored at $4 \pm 2{ }^{\circ} \mathrm{C}$.

\section{Analytical methods}

Particle sizing. Mastersizer 2000 (Malvern Instruments Ltd, UK) was used to determine the size of fat particles dispersed in water and the droplet size of the water-continuous emulsions. Refractive indices 1.54 and 1.33 were used for tripalmitin particles and liquid-liquid emulsions, respectively. The droplet size of the oil-continuous emulsions was measured using a pulsed field gradient NMR (Bruker Minispec, UK), as previously reported by Frasch-Melnik et al. ${ }^{5}$ Samples were prepared in at least duplicate and reported as an average of three measurements.

Thermal analysis. Thermal behaviour of the particle dispersions in water were determined by a Differential Scanning Calorimetry (DSC) method using a Perkin-Elmer 7 device (Perkin-Elmer, UK). The temperature was cycled between 5 and $80^{\circ} \mathrm{C}$ at a rate of $10^{\circ} \mathrm{C} \mathrm{min}^{-1}$, with an empty pan as a reference. Due to a low amount of the crystalline fat present in the oil-continuous emulsions, their thermal properties were determined using a micro-calorimeter Setaram (Setaram Instrumentation, France). The temperature was cycled between 5 and $80{ }^{\circ} \mathrm{C}$ (at a rate of $1{ }^{\circ} \mathrm{C} \mathrm{min}^{-1}$ ) against a sunflower oil reference.

Imaging. Images of the tripalmitin particle dispersions and emulsions were obtained after their preparation and regularly during storage using a light microscope (Brunel Microscopes, UK with a Canon video camera). Emulsions were also visualised using a Phillips XL30 FEG Cryo Scanning Electron Microscope equipped with a Gatan low temperature unit. A single drop of emulsion was placed on an analysis slide and dipped 
into nitrogen at $-198{ }^{\circ} \mathrm{C}$. This slide was then inserted directly into a preparation chamber at $-180{ }^{\circ} \mathrm{C}$ where it was fractured and subsequently etched for $5 \mathrm{~min}$ at $-90^{\circ} \mathrm{C}$. The surface was coated in gold and then imaged in the SEM at $-130^{\circ} \mathrm{C}$.

\section{Results and discussion}

\section{Production and characterisation of solid lipid particles}

Size and morphology. Water dispersions of solid lipid particles $(5 \%)$ were produced via a hot sonication route. The emulsifier concentration for: (i) effective stabilisation of a tripalmitin emulsion (i.e. molten fat during the emulsification) and (ii) subsequent solid particle production during cooling and storage (i.e. during crystal seeding, growth and polymorphic transitions) was investigated. Tween 20 at different concentrations $(1,2,4$ and $5 \%)$ was added to the water phase before the sonication. Particle size distributions were then measured $24 \mathrm{~h}$ after their preparation, and are given in Fig. 1.

Fig. 1 shows that TP average particle size decreases from $\sim 25 \mu \mathrm{m}$ to $\sim 150 \mathrm{~nm}$ as the concentration of the surfactant increases from 1 to $4 \%$; it then remains constant on a further Tween 20 increase to 5\%. Fig. 2A shows that discrete particles as well as larger aggregates coexist in the formulation with $1 \%$ Tween 20, which explains the increase in the measured average particle size with decreasing concentration of Tween 20. During cooling of the molten emulsion, gelation ${ }^{21}$ of formulations containing 1 and 2\% Tween 20 was observed (weak gels, reversible on stirring). On the contrary, formulations with 4 and 5\% Tween 20 were fully liquid, milky with a "bluish" tint. This suggests that below 4\% Tween 20 there was insufficient surfactant (Tween 20) to fully cover the interfacial area, consequently leading to coalescence of liquid tripalmitin prior to crystallisation. In addition, due to incomplete coverage and/ or an insufficient concentration of free surfactant molecules in the bulk, the tripalmitin droplets could have aggregated during the cooling process, when crystal growth or/and polymorphic transformations caused a further increase in the interfacial area. ${ }^{21}$ Such aggregation could result in gelling (i.e. attractive interactions between the hydrophobic chains of tri-

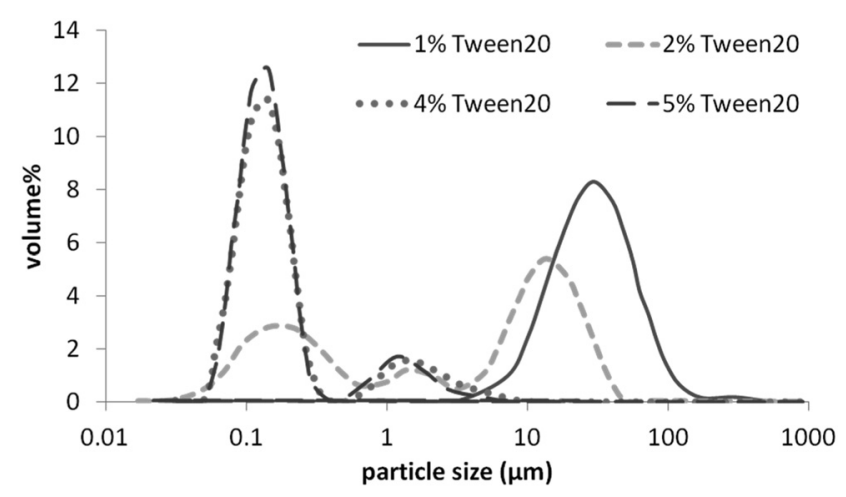

Fig. 1 Particle size distribution of solid lipid dispersions; 5\% tripalmitin in water, stabilised with 1, 2, 4 and $5 \%$ Tween 20.

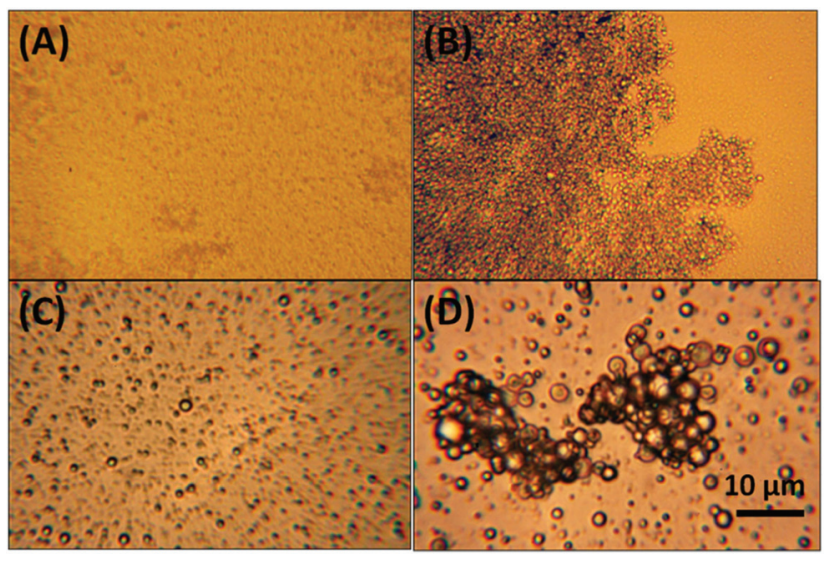

Fig. 2 Micrographs of solid lipid dispersions; $5 \%$ tripalmitin in water, stabilised with: (A) $1 \%$ Tween 20, (B) $1 \%$ PGPR, (C) $5 \%$ WPI and (D) no emulsifier.

glyceride crystals, reversible on stirring) or partial coalescence of solid fat particles, where the crystal growing in one particle can pierce the neighbouring one, bridging them into an irreversible aggregate. The case of the partial coalescence is supported by observations of Westesen \& Siekmann, ${ }^{21}$ who, using a TEM technique, visualised needle-shaped tripalmitin crystals. It has also been reported ${ }^{22}$ that polymorphic transitions in fat particles lead to a change in the crystal shape, subsequent increase in the interfacial area, and particle aggregation even in the presence of a relatively high concentration of Tween 20. This is likely to be caused by the relatively poor steric properties of a low molecular weight surfactant such as Tween 20. As a result, particle-particle contact during (re)crystallisation, or particle collisions, may potentially lead to aggregation. In order to overcome these limitations, a range of food grade emulsifiers were investigated for their potential to stabilise tripalmitin fat particles in aqueous dispersion. Soy lecithin, WPI (globular and heat-sensitive protein), PGPR (oil-soluble emulsifier) and their mixtures with low molecular weight Tween 20 (i.e. lecithin-Tween 20 and WPI-Tween 20) were investigated with the goal of providing: (i) fast interfacial coverage during emulsification, and (ii) a thick interfacial film that imparts post production stability. Solid fat particles without any emulsifier were also investigated.

Without an emulsifier present during emulsification, a 5\% tripalmitin-in-water system was milky white with no macroscopic phase separation or bulk gelation occurring during cooling of the molten emulsion. The particle size distribution of the dispersion was complex (Fig. 3A), with three populations ca. 0.15, 2 and $25 \mu \mathrm{m}$. Microscopic images showed near-spherical, partially flocculated particles (Fig. 2D); however, a few bigger "lumps" of fat (1-2 mm) were also present. To investigate the potential of breaking down these flocculated crystals, the suspension was treated via a mild sonication process (to avoid excessive heat production); however, no significant reduction in particle size was achieved. To further reduce the size of solid fat particles in water, a cold high-pressure 

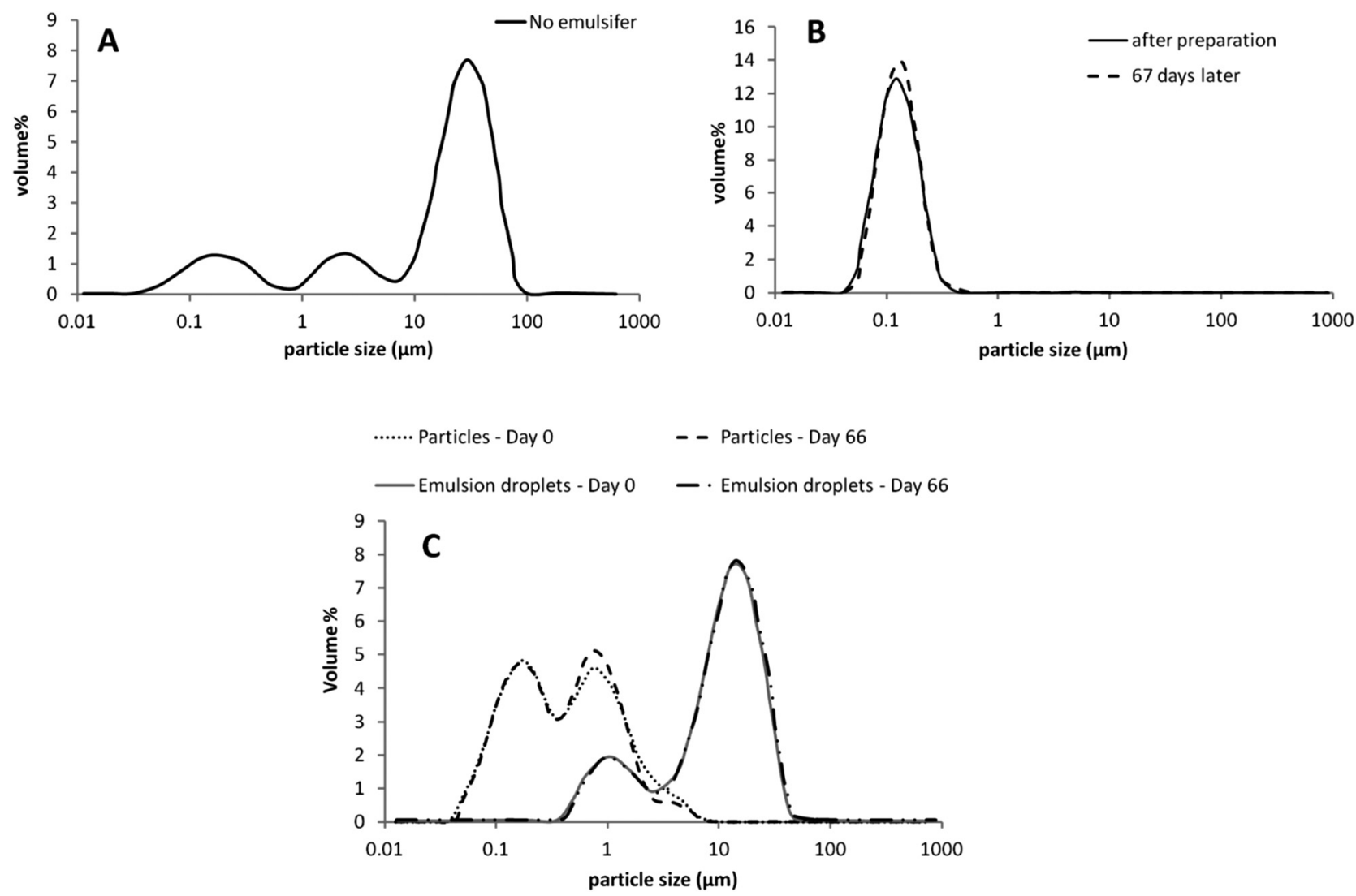

Fig. 3 Particle size distribution measured over the storage period. Tripalmitin particles with: (A) no emulsifier, (B) $5 \%$ lecithin-Tween 20 (1:1) and (C) $5 \%$ WPI. Also in (C) droplet size of O/W emulsions stabilised with tripalmitin-WPI particles.

homogenisation (NS 1001L Panda) was also performed. However, the hydrophobic nature of these particles caused a blockage inside the processing equipment. The hydrophobic character of the particles also manifested in their propensity for adsorption at air-water interface, resulting in formation of a leathery wrinkled film. A similar film of particles was observed by Do et al. for water-insoluble ethyl cellulose particles investigated for use as viscosity modifiers in low fat chocolate $^{23}$ The fact an emulsifier was not required to produce a stable dispersion of fat particles in water is perhaps surprising, as one could expect coalescence of fat during sonication and cooling. The stability of fat particles in water could be enhanced by surface-active impurities (e.g. monoglycerides) present in the tripalmitin, which might cover the interface during emulsification and subsequent storage. Additionally, during the cooling step, $\alpha$ crystals of tripalmitin that form first have been reported ${ }^{16}$ to be more polar than the $\beta$ crystals. This increase in polarity may ensure that the energy penalty for fat particles in the dispersed state is smaller due to their relatively increased propensity for water.

When soy lecithin $(5 \%)$ was used to stabilise $5 \%$ tripalmitin-in-water, the particle dispersion gelled on cooling and, as such, a laser diffraction particle size measurement was impossible. Gelation in the lecithin-tripalmitin system can be explained by the specific behaviour of lecithin in water, i.e. formation of lamellar phases, which exhibit lower mobility than highly dynamic surfactant micelles. Therefore, when the fat dispersion was cooled down after the emulsification, there was no shearing force to disrupt liquid crystals of lecithin and so its adsorption to the newly formed interfaces (during crystal growth on cooling) was slow. We have shown, on the example with the fat particles with no emulsifier (Fig. 3A), that an uncovered interface does not necessarily lead to particle aggregation and gelation of bulk tripalmitin. Hence, gelation of the tripalmitin dispersion in the presence of lecithin is more likely caused by an attractive particle interactions induced by the phospholipid. Lecithin has a complex composition and on the molecular level its structure is somewhat similar to that of a triglyceride. $^{21}$ By being incorporated into the tripalmitin network, lecithin may bridge between the neighbouring particles thus leading to aggregation. We have shown (Fig. 3B) that particle aggregation in the formulation with lecithin can be avoided by using a co-emulsifier, a small molecular surfactant (Tween 20, 1:1 with lecithin). Such formulation design ensures production of small tripalmitin particles $(120 \mathrm{~nm}$, Fig. 3B) resulting from fast adsorption of Tween 20 via its highly dynamic micellar structure. Moreover, fast coverage of the interface may also limit the bridging effect of the adsorbing lecithin, which nevertheless introduces a steric interfacial barrier against crystals protruding towards the neighbouring 
particles. As a result, fat dispersions stabilised by the mixture of a lecithin and Tween 20 were stable over the 9 week observation period.

Producing solid tripalmitin particles with 5\% WPI resulted in a bimodal particle size distribution (Fig. 3C), with two size populations, $c a$. 150 and $700 \mathrm{~nm}$, which did not change significantly over the storage period of 9 weeks. The bimodal size distribution could be caused by a heat-induced partial denaturation and unfolding of the protein (during sonication), ${ }^{23}$ subsequently lead to aggregation of such protein-stabilised fat particles. Microscopic images (Fig. 2C), however, showed no apparent flocs. Moreover, partial coalescence or gelling of particles during cooling is likely to be hindered by a relatively thick layer of the adsorbed protein and electrostatic repulsions induced by them. Instead, the reason behind the bimodal size distribution is more likely as result of coalescence of the liquid fat droplets during emulsification. This may be result of the high molecular weight of WPI $\left(18 \mathrm{~kg} \mathrm{~mol}^{-1}\right)$ and the fact in the region $\mathrm{pH}=5-8 \mathrm{WPI}$ exists as a dimer. ${ }^{24}$

WPI was also combined with a co-emulsifier (Tween 20) in a $1: 1$ ratio and used to stabilise tripalmitin dispersions. However, in this case, the fat phase separated from water during cooling and stayed as a solid layer on the top of the bulk water phase. A displacement of WPI by Tween 20 is highly questionable owing to a multiple point protein anchoring at the interface. At the moment, the exact reason for this behaviour is unknown but is probably governed by the nature of interaction between WPI and tripalmitin.

Tripalmitin suspensions were also stabilised with $1 \%$ PGPR. These dispersions were mildly gelled (thus laser diffraction particle size data could not be obtained) and the microscopic images showed relatively small particles of uniform size (Fig. 2B). Gelation is often caused by an insufficient surfactant concentration in the system.

Thermal behaviour. DSC heating and cooling curves for bulk tripalmitin and tripalmitin aqueous dispersions (with and without emulsifiers) were obtained to evaluate whether these systems exhibit polymorphism. Fig. 4 and 5 show normalised melting and re-crystallisation curves (respectively) for tripalmi-

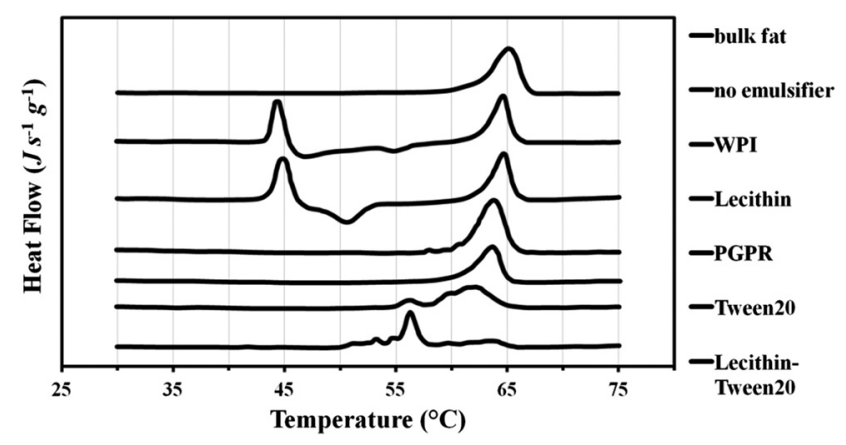

Fig. 4 DSC melting curves of bulk tripalmitin and particle dispersions in water. Scanning rates of $10{ }^{\circ} \mathrm{C} \mathrm{min}-1$ were applied and empty pan was used as a reference. The curves are displaced along the $y$-axis for easier visualisation.

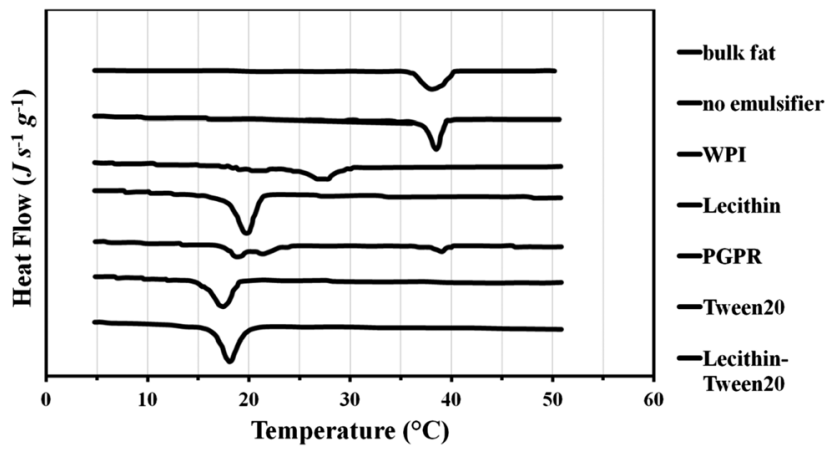

Fig. 5 DSC crystallisation curves of bulk tripalmitin and particle dispersions in water. Scanning rates of $10^{\circ} \mathrm{C} \mathrm{min}^{-1}$ were applied and empty pan was used as a reference. The curves are displaced along the $y$-axis for better visualisation.

tin particles with and without the emulsifiers, as well as bulk tripalmitin.

The peak melting temperature of bulk tripalmitin was $65^{\circ} \mathrm{C}$ (Fig. 4); this is within the range of the values reported by Helgason et al., Bunjes et al., and Knoester et al.: ${ }^{22,25,26} 63,64$ and $66^{\circ} \mathrm{C}$, respectively. The difference between the reported values is most likely a consequence of varying tripalmitin purity across the experiments. The melting curves in Fig. 4 show that when no emulsifier is present to stabilise the tripalmitin in dispersion, first the meta-stable $\alpha$ crystalline form melts at $44{ }^{\circ} \mathrm{C}\left(45^{\circ} \mathrm{C}\right.$ in Helgason et $\left.a .^{22}\right)$, followed by an exothermic peak at $55{ }^{\circ} \mathrm{C}$ (i.e. recrystallisation into a more stable $\beta^{\prime}$ or $\beta$ ) and then melting of a stable $\beta$ form at $65{ }^{\circ} \mathrm{C}$. This is identical to the melting temperature of a $\beta$ form in the bulk tripalmitin. Similar melting behaviour was exhibited by the WPI stabilised tripalmitin particles; however, the recrystallisation peak ( $\alpha$ to $\beta^{\prime}$ or $\beta$ ) occurred at $50{ }^{\circ} \mathrm{C}$. The $\alpha$ crystals were no longer present in formulations containing lecithin or PGPR, where one endothermic peak at $64{ }^{\circ} \mathrm{C}$ indicated the presence of the stable $\beta$ crystals. This suggests that that both lecithin and PGPR promote crystal lattice zone refinement, facilitating crystal movements and thus their transition to the most energetically favourable state. This can be explained by the fact that both emulsifiers have large protruding hydrophobic tails, which sufficiently penetrate into the particle's fat matrix. Bulky hydrophobic tails provide large spacing between fat molecules, creating grain boundaries and easing molecular reorganisation within the particle. Alternatively, the molecular similarity of the emulsifier chains to the fat molecules (such as lecithin to tripalmitin) may mean that they initiate (or promote) crystallisation of the more stable crystal form. Contrary to the lecithin and PGPR, WPI may adsorb on the crystal surface interface rather than penetrate into the fat crystal; this is because a globular protein first adsorbs at the interface and then unfolds over time, revealing its hydrophobic moieties. However, quick fat solidification may restrict protein unfolding at the interface, limiting the number of hydrophobic moieties penetrating the fat phase. As a result, two mechanisms are possible. Firstly, due to its position at the interface, WPI does 
not enter the fat matrix at all and thus the system behaves like an emulsifier free dispersion (thus unaided and slow polymorphic transformation). Secondly, the viscoelastic membrane formed by the interfacial protein, prevents the crystal movements, slowing down the polymorphic transformations. It has been postulated by Helgason et al. ${ }^{22}$ that close packing of surfactant tails may form a relatively rigid "shell" surrounding the lipid particle, which then restricts the molecular motions within the fat matrix.

When Tween 20 and its mixture with the lecithin were employed, there was a general decrease in a melting temperature of the most stable crystal form. Fig. 4 shows that Tween 20 on its own caused the crystal structure to melt first at $56^{\circ} \mathrm{C}$ (most likely $\beta^{\prime}$ ) followed by a larger peak at $62{ }^{\circ} \mathrm{C}(\beta)$. When lecithin was added to Tween 20 , the fat melting curve is of multiple peaks with maxima at $48{ }^{\circ} \mathrm{C}, 56^{\circ} \mathrm{C}$ (biggest peak) and $63^{\circ} \mathrm{C}$. There are several possible explanations for such thermal behaviour. Firstly, as suggested by Helgason $e t$ al. ${ }^{22}$ the emulsifiers could significantly slow down or even stop the polymorphic transition at the metastable $\beta^{\prime}$ form (melting at $56{ }^{\circ} \mathrm{C}$ ). Through specific interactions occuring at the interface (leading to, for instance, an increase in viscoelasticity) crystal movement can be transiently hindered by the emulsifier film present. An additional argument supporting the presence of metastable crystals comes from the fact that the melting peaks are very broad, suggesting a high degree of imperfections (grain boundaries) in the crystal lattice ${ }^{27}$ typical for lower melting crystalline forms. Secondly, the depression of the melting point of the stable $\beta$ crystals (to $56{ }^{\circ} \mathrm{C}$ ) could be caused by very small sizes of fat particles. ${ }^{27}$ Such particles have very high surface curvatures, which increases their solubility and thus lowers the melting temperature. Such dampening of the melting temperature does not occur when larger particles, for example, those with no emulsifier or stabilised with WPI and lecithin (Fig. 4). The latter explanation seems more likely, as the attempts to temper the particle crystal matrix, by keeping it (up to $2 \mathrm{~h}$ ) at the temperature slightly above the melting temperature, did not result in further polymorphic transformation.

DSC curves for crystallisation of the solid lipid particles are given in Fig. 5. The crystallisation of bulk tripalmitin, via formation of $\alpha$ crystals, took place at $39^{\circ} \mathrm{C}$, which is between $37^{\circ} \mathrm{C}$ reported by Helgason et al. and $42{ }^{\circ} \mathrm{C}$ reported by Bunjes et al. A similar crystallisation temperature of $38{ }^{\circ} \mathrm{C}$ was observed for the fat dispersion in the absence of the emulsifiers. This would be expected as prior heating of the sample (i.e. the melting curve shown in Fig. 4) causes fat coalescence, phase separation and thus subsequent bulk fat crystallisation behaviour. Fig. 5 shows that Tween 20, lecithin and the corresponding mixture caused a significant decrease in the recrystallisation temperature to 17,20 and $18{ }^{\circ} \mathrm{C}$, respectively. This is due to the requirement for substantial super-cooling of particles with a smaller average size. Using WPI, recrystallisation of tripalmitin occurred at $27^{\circ} \mathrm{C}$ due to larger sizes of the particles (up to $700 \mathrm{~nm}$, Fig. 3) and thus a reduced degree of supercooling required. Recrystallisation of tripalmitin particles stabilised with PGPR show a first small peak at $39{ }^{\circ} \mathrm{C}$ followed by a multi-peak with a maximum at $19^{\circ} \mathrm{C}$. This demonstrates that PGPR (being an oil soluble emulsifier) was not particularly efficient at stabilising the molten tripalmitin dispersion and/ or the PGPR concentration employed was too low. This could lead to coalescence of a significant number of liquid fat droplets, which then recrystallised in a bulk fat manner (at $39^{\circ} \mathrm{C}$ ), while the rest of stable particles recrystallised at the temperature $\left(\right.$ at $19{ }^{\circ} \mathrm{C}$ ) similar to the dispersions with lecithin and Tween 20, where no coalescence occurred.

Thermal analysis was repeated on all samples after one week and also one year of storage and no significant change in the thermal behaviour was observed. This means that polymorphic transformations facilitated by certain emulsifiers happen relatively fast (up to $24 \mathrm{~h}$ ). However, when such emulsifiers are not present or do not penetrate the fat network (i.e. WPI), the metastable forms of tripalmitin crystals exist for at least a year.

\section{Production \& characterisation of emulsions}

Size and morphology. Tripalmitin particles in water, stabilised with or without the addition of the emulsifiers, were used without further processing (e.g. no dilution) to produce emulsions. Apart from the particles with WPI, which formed O/W emulsions (with $20 \%$ sunflower oil), all other particle formulations showed preference for forming W/O emulsions. Emulsions of $20 \%$ water (with fat particles dispersed within) in $80 \%$ sunflower oil were produced and analysed over time.

The droplet size evolution of $\mathrm{W} / \mathrm{O}$ emulsions with time, measured using a NMR, is given in Fig. 6. It shows that the emulsions stabilised with tripalmitin particles without the emulsifiers, with Tween 20, and with PGPR had similar droplet sizes $(c a .15 \mu \mathrm{m})$ and did not change significantly over the storage period. All emulsions, however, as expected, displayed sedimentation on storage, with a distinct layer of clear oil on the top of the storage vessel. When particles were stabilised with a lecithin-Tween 20 mixture, the resulting emulsion

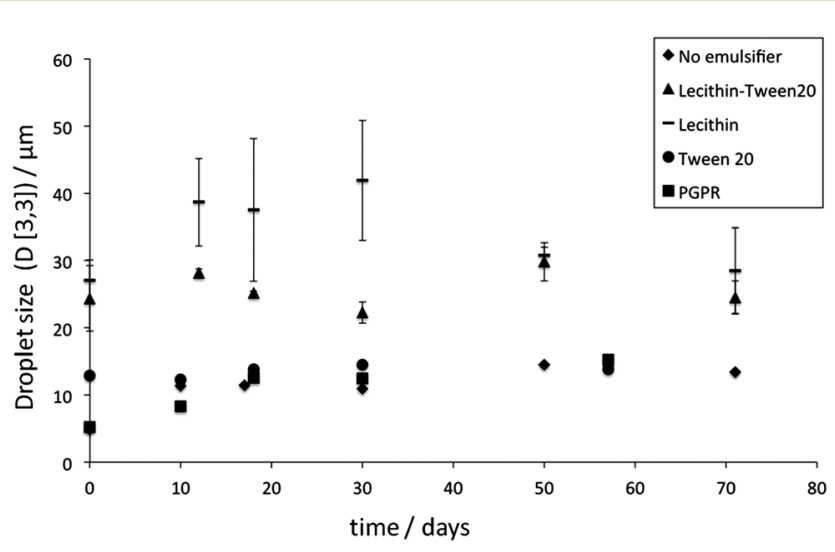

Fig. 6 W/O emulsions stabilised with fat particles; droplet size evolution with time. Where error bars cannot be visualised they are covered by the symbol. 
had a larger average droplet size $(25-30 \mu \mathrm{m})$ and were also stable of the storage period. Optical and SEM micrographs of the emulsion with the lecithin-Tween 20 stabilised tripalmitin (Fig. 7B and 8F) show spherical water droplets covered with particles of different sizes. The emulsions stabilised with tripalmitin-lecithin particles visibly flocculated and after the initial 2 weeks of storage, large water droplets sedimented on the bottom of the container. This was accompanied by an increase in the droplet size from $\sim 27 \mu \mathrm{m}$ (after preparation) to $\sim 42 \mu \mathrm{m}$ (day 30) followed by a decrease to $\sim 29 \mu \mathrm{m}$ (day 72) (Fig. 6).

The latter droplet size is a result of "free water" in the sample. "Free water" is any water compartment that is not encapsulated within the $\sim 50 \mu \mathrm{m}$ diameter droplet. Therefore, in water droplets larger than $\sim 50 \mu \mathrm{m}$ the instrument detects an unrestricted diffusion and identifies them as "free water". As a result of such a limitation, the average calculated droplet size is reduced. The droplet size data shows (Fig. 6), that even though the particles stabilised with Tween 20 and a mixture of lecithin and Tween 20 are of a similar size (i.e. $130 \mathrm{~nm}$, Fig. 1 and $3 \mathrm{~B}$ ), the emulsion obtained with the tripalmitin-Tween 20 particles had markedly smaller droplets. This means that: (i) not only the size of the particles determines the curvature of the interface, but also their surface properties and/or (ii) a higher concentration of free (unbounded to fat particles) Tween 20 molecules in the system with tripalmitin. Tween 20 allows for a faster decrease in the interfacial tension between sunflower oil and water during the emulsification.

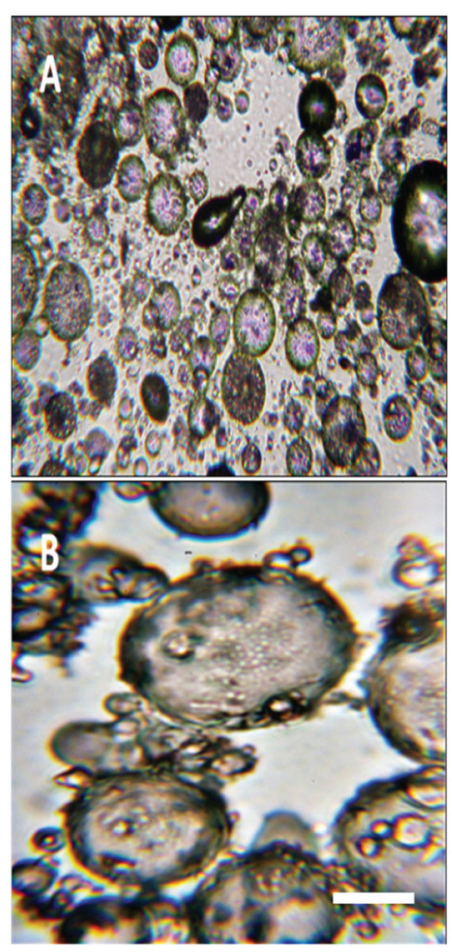

Fig. 7 Micrographs of: (A) O/W emulsion stabilised with fat particles made with $5 \%$ WPI and (B) W/O emulsion stabilised with fat particles made with lecithin-Tween 20 mixture (1:1). Scale bar $20 \mu \mathrm{m}$.
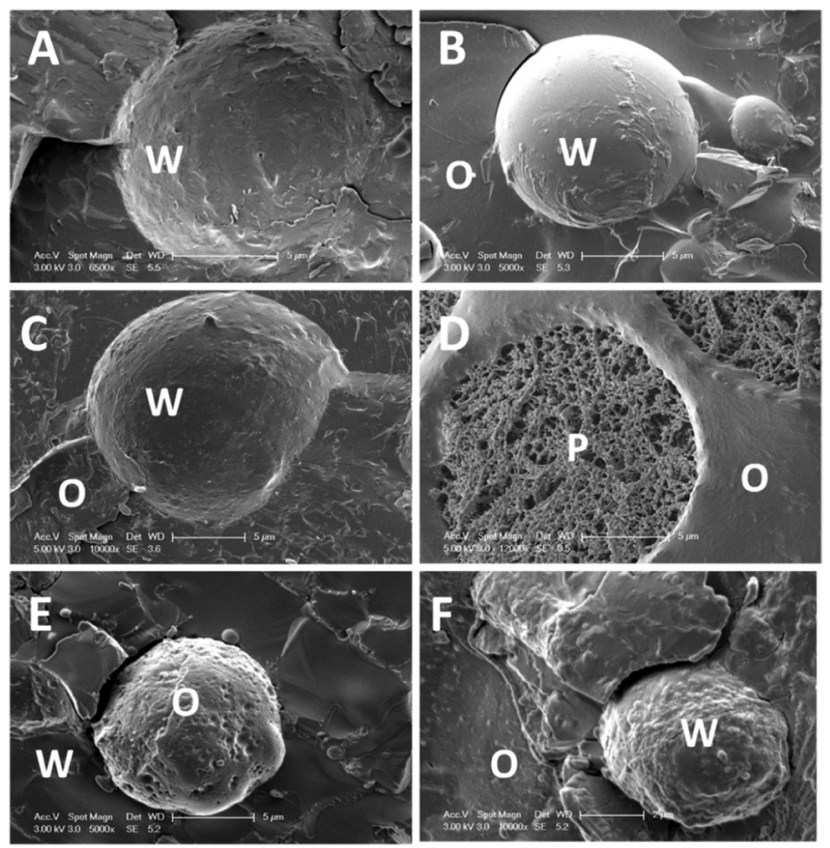

Fig. 8 SEM images of emulsions stabilised with fat particles: (A) W/O emulsion, particles with no emulsifier, (B) W/O emulsion, particles with 1\% PGPR, (C) W/O emulsion, particles with $5 \%$ Tween 20 , (D) W/O emulsion, particles with $5 \%$ Tween 20 , after water removal, (E) O/W emulsion, particles with $5 \% \mathrm{WPI}$ and (F) W/O emulsion, particles with $5 \%$ lecithinTween 20 mixture (1: 1). W-water, O-oil, P-particles.

Tripalmitin particles produced with WPI showed a preference for the formation of $\mathrm{O} / \mathrm{W}$ emulsions. This is most probably due to their hydrophilic character, which originates from the $\alpha$ crystalline form of tripalmitin (Fig. 4) and a relatively thick layer of the protein at the particle interface. The droplet size distribution of such emulsions measured directly after their preparation and during storage, is shown in Fig. 3C. It is evident that the emulsion is stable against coalescence over a period of 50 days. Moreover, microscopic analyses (Fig. 7A and 8E) show that the oil droplets are covered by particles, which suggests the Pickering stabilisation mechanism. We propose that the produced emulsions are stabilised by Pickering particles rather than purely emulsifiers used for the particle preparation. This is supported by the fact that: i. the emulsion stabilised with the tripalmitin particles without any emulsifier was stable, ii. all emulsifiers used (except PGPR) are water-soluble and do not stabilise oil-continuous emulsions. For example, 5\% Tween 20 in the formulation with water and oil (1:4 ratio) caused a phase inversion to a water-continuous emulsion and iii. Microscopic analyses showed emulsion interfaces were covered by particles.

Thermal behaviour. In order to study a thermal behaviour of the crystalline fat used to stabilise emulsions, differential scanning calorimetry was performed on all emulsion samples. The melting curves of W/O emulsions were obtained using a $\mu$-DSC and are shown in Fig. 9. It can be seen that the tripalmitin-particles-no emulsifier-stabilised emulsions began melting at 


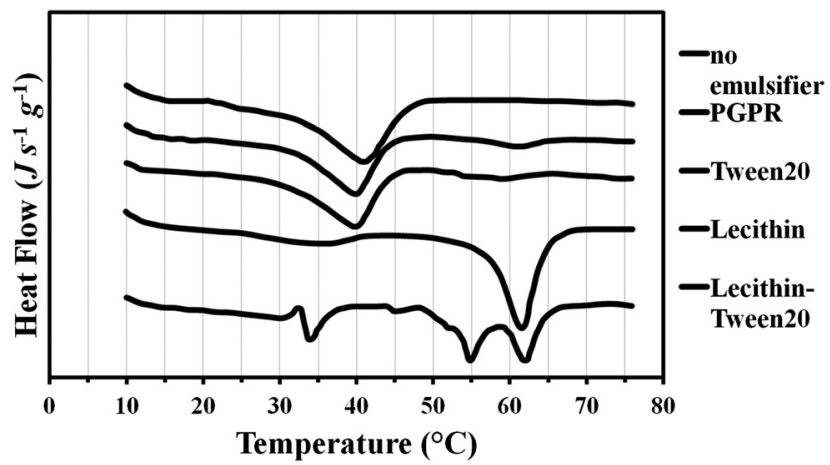

Fig. $9 \mu$-DSC melting curves of W/O emulsions stabilised with fat particles made with or without emulsifiers. Scanning rates of $1{ }^{\circ} \mathrm{C} \mathrm{min}^{-1}$ were applied and oil-filled pan was used as a reference. The curves are displaced along the $y$-axis for better visualisation.

$31{ }^{\circ} \mathrm{C}$ (onset temp.) with a peak maximum at $41{ }^{\circ} \mathrm{C}$. This is significantly lower than for the tripalmitin particles in dispersion $\left(65{ }^{\circ} \mathrm{C}\right.$ in Fig. 4). A similar shift of the melting temperature and an overall broadening of the peak has been reported by Norton et al. ${ }^{28}$ and was attributed to a solubility of tripalmitin in the liquid oil (triolein in that case). When the emulsifier stabilised tripalmitin particles were used to make emulsions, the melting behaviour of these emulsions was different. The emulsions stabilised with the tripalmitin-PGPR and tripalmitin-Tween 20 particles both show the first broad melting peak at $40{ }^{\circ} \mathrm{C}$ and then a smaller peak at 61 and $59{ }^{\circ} \mathrm{C}$, respectively (Fig. 9).

When tripalmitin-lecithin particles were used for emulsion stabilisation, the low temperature peak was small and broad and, as such, the melting temperature was difficult to determine. The broad peak was followed by melting of crystalline material at $62{ }^{\circ} \mathrm{C}$ (represented by a single peak in Fig. 9) similar to the melting temperature of the $\beta$ crystals in the respective particle dispersion $\left(64^{\circ} \mathrm{C}\right.$ shown in Fig. 4). When particles were prepared with the lecithin-Tween 20 mixture, the melting curve of the emulsion they stabilise was very complex (Fig. 9), with multiple peaks across the whole range of scanning temperatures (broad peak below $31{ }^{\circ} \mathrm{C}$ followed by peaks at $34,44,55$ and $61^{\circ} \mathrm{C}$ ). These peak temperatures correspond to the melting of the respective particle dispersion in water (48, 56 and $63{ }^{\circ} \mathrm{C}$, Fig. 4). Thermal properties of the $\mathrm{O} / \mathrm{W}$ emulsions stabilised with the tripalmitin-WPI particles were identical to these of a tripalmitin-WPI dispersion (Fig. 4). This is caused by the large amount of free fat particles residing in the water-continuous phase. Melting of the bulk particles superimposes the melting of the interfacial particles, thus it is impossible to discriminate them.

The explanations for the differences between the emulsions stabilised with tripalmitin combined or not with an emulsifier, are as follows. When no emulsifier is used to stabilise fat particles, they become solubilised by the liquid oil at the emulsion's interface, which is manifested by reduction of the tripalmitin melting temperature (Fig. 9). The solubi- lisation process also promotes rearrangements of the fat molecules at the interface and consequently the crystals sinter into a shell-like structure ${ }^{5}$ around the water droplet (Fig. 8A).

A similar mechanism occurs when the tripalmitin particles are covered with Tween 20 and PGPR, which both facilitate zone refinement of the fat crystal network, promoting its reorganisation into the most energetically favourable state (Fig. 4). A relatively small hydrophilic group of the oil-soluble PGPR does not significantly influence the polarity of the particle surface. And so the particles stay hydrophobic, become gradually solubilised by the liquid oil at the emulsion interface, and then sinter to form a relatively smooth fat shell (Fig. 8B). This is marginally less so for Tween 20 (Fig. 8C), which has larger hydrophilic head groups, making the particles more polar and thus a portion of such particles stays within the water phase. This is confirmed by a small melting peak around $59{ }^{\circ} \mathrm{C}$ (Fig. 9) and microscopic observations (Fig. 8D). Manipulation of the pressure and temperature during the SEM imaging, revealed the inside of the water droplet, shown in Fig. 8D. Forcing the water out of the system exposed a network of Tween 20-stabilised fat particles, suggesting their affinity for the polar phase. The above data suggests that, the larger the surfactant hydrophilic head group is (Tween $20>$ PGPR), the bigger the steric energy barrier against particles sintering at the interface. In emulsions, highly dynamic, non-ionic surfactants do not usually form strong repulsive interactions at the interface, hence do not create a significant steric barrier against the emulsion instability. However, when surfactant molecules at the interface are immobilised within the fat matrix, their ability to provide steric protection against aggregation is increased.

We propose that lecithin molecules either promote zone refinement of the fat matrix by aiding the polymorphic transitions or, due to a similarity in the molecular structure, facilitate fat crystallisation in the most stable crystal form (Fig. 4). Thermal characterisation of the tripalmitin-lecithin stabilised W/O emulsions, reveals large amounts of the crystalline material with the similar properties to these of the respective particle dispersion (melting at $62^{\circ} \mathrm{C}$ ). This suggests a high particle affinity for water and thus small number of particles in contact with the liquid oil. The reason behind this could be that the adsorbed lecithin molecules make the fat surface more polar ${ }^{29}$ and so the fat solubility in the liquid oil decreases. This potential increase in the polar behaviour of the tripalmitin-lecithin particles results in the particles preference to reside within the aqueous phase, followed by the instability of the oil-continuous emulsion. This emulsion breakdown during pre-SEM-imaging preparation made it impossible to visualise the sample. When Tween 20 and lecithin were used in conjunction, the reduced amount of the lecithin in the overall formulation resulted in a decrease in particle polarity. They still mainly reside in the water phase (demonstrated by a presence of the high melting crystals in Fig. 9), but there is also a significant percentage of particles adsorbed at the interface and so in the contact with the liquid oil (low melting 
peaks below $35^{\circ} \mathrm{C}$ ). SEM images (Fig. $8 \mathrm{~F}$ ) reveal a rough interface, suggesting that interfacial crystal sintering is limited, most likely due to steric hindrance of large head groups of lecithin and Tween 20.

WPI, as discussed earlier, does not significantly penetrate into the tripalmitin particles. Through formation of a thick protein interfacial membrane and combined with the $\alpha$ form of the fat crystals (Fig. 4), the particles exhibit more hydrophilic character and thus stabilise water-continuous emulsions. When the particles are in contact with the liquid oil (i.e. in emulsion) their visco-elastic protein layer provides a sufficient steric and electrostatic barrier against sintering. This is confirmed by the SEM images of $\mathrm{O} / \mathrm{W}$ emulsion stabilised with the tripalmitin-WPI particles (Fig. 8E). Except for the tripalmitin-PGPR stabilised, all other emulsions phase separated when heated up to $80^{\circ} \mathrm{C}$ during the DSC cycle, and as such, we decided recrystallisation was not relevant.

\section{Conclusions}

We have shown that by using a variety of food grade emulsifiers, tripalmitin particles with different characteristics with respect to their emulsion-forming tendencies can be produced. We propose that two mechanisms are involved. Firstly, emulsifiers can affect the rate and/or the extent of fat polymorphic transitions and result in a formation of fat crystals with modified polarity. Secondly, adsorption of an emulsifier to the particle interface may also serve to modify its surface properties. We propose that the polarity of the fat particles increases in the following order: no emulsifier $<$ PGPR $<$ Tween $20<$ lecithin + Tween $20<$ lecithin $<$ WPI. As a consequence, highly polar tripalmitin particles produced with WPI stabilised watercontinuous emulsions; particles with lecithin formed unstable oil-continuous emulsions; and with lecithin-Tween 20 mixtures, an oil-continuous emulsion was produced with a comparatively large drop size. Tripalmitin particles prepared with Tween 20, PGPR, or no emulsifier, formed stable oil-continuous emulsions with sintered fat shells around the water droplets. Further analytical approaches to study the formation of sintered shells would be a worthwhile extension to this work. Emulsion droplets with defined interfacial permeability may be produced using this approach and this could prove useful for controlled delivery of food/pharmaceutical ingredients. However, further understanding of the sintering mechanism is required, both in terms of chemistry and process.

There is still much to learn about designing new edible particles. Such particles have enormous appeal in foods and other emulsion-based products, with key technical drivers including improvements in product shelf life and/or controlled and targeted release of functional ingredients. We have shown how to produce emulsions stabilised via a Pickering mechanism, wherein the Pickering particle functionality (towards its w/o or $\mathrm{o} / \mathrm{w}$ emulsion forming potential) is controlled by the emulsifier chosen in particle manufacture.

\section{Acknowledgements}

We would like to thank the EPSRC for financial support as well as Dr Phil Taylor for useful discussions.

\section{References}

1 B. P. Binks, Curr. Opin. Colloid Interface Sci., 2002, 7, 21 .

2 B. S. Murray, K. Durga, A. Yusoff and S. D. Stoyanov, Food Hydrocolloids, 2011, 25, 627.

3 R. Pichot, F. Spyropoulos and I. T. Norton, J. Colloid Interface Sci., 2010, 352, 128.

4 M. Kargar, K. Fayazmanesh and M. Alavi, J. Colloid Interface Sci., 2012, 366, 209.

5 S. Frasch-Melnik, I. T. Norton and F. Spyropoulos, J. Food Eng., 2010, 98, 437.

6 R. Pichot, F. Spyropoulos and I. T. Norton, J. Colloid Interface Sci., 2009, 329, 284-291.

7 B. P. Binks and A. Rocher, J. Colloid Interface Sci., 2009, 335, 94-104.

8 W. Z. Zhou, J. Cao, W. C. Liu and S. Stoyanov, Angew. Chem., Int. Ed., 2009, 48, 378-381.

9 A. L. Cambell, S. D. Stoyanov and V. N. Paunov, Soft Matter, 2009, 5, 1019-1023.

10 S. Schmitt and S. L. Turgeon, Adv. Colloid Interface Sci., 2011, 167, 63-70.

11 A. Timgren, M. Rayner, M. Sjoo and P. Dejmek, Procedia Food Sci., 2011, 1, 95-103.

12 D. E. Tambe and M. M. Sharma, Adv. Colloid Interface Sci., 1994, 52, 1-63.

13 B. P. Binks, J. A. Rodrigues and W. J. Frith, Langmuir, 2007, 23, 3626-3636.

14 B. P. Binks and J. A. Rodrigues, Langmuir, 2007, 23, 74367439.

15 C. Vashisth, C. P. Whitby, D. Fornasiero and J. Ralston, J. Colloid Interface Sci., 2010, 349, 537-543.

16 D. Johansson, B. Bergenstahl and E. Lundgren, J. Am. Oil Chem. Soc., 1995, 72, 921-931.

17 J. W. Mullin, Crystallisation, Butterworth-Heineman, 3 edn, 1993.

18 S. Ghosh, T. Tran and D. Rousseau, Langmuir, 2011, 27, 6589-6597.

19 S. M. Hodge and D. Rousseau, J. Am. Oil Chem. Soc., 2005, 82, 159-164.

20 N. Garti, H. Binyamin and A. Aserin, J. Am. Oil Chem. Soc., 1998, 75, 1825-1831.

21 K. Westesen and B. Siekmann, Int. J. Pharm., 1997, 151, 35-45.

22 T. Helgason, T. S. Awad, K. Kristbergsson, D. J. McClements and J. Weiss, J. Colloid Interface Sci., 2009, 334, 75-81.

23 T. A. L. Do, J. Vieira, J. M. Hargreaves, J. R. Mitchell and B. Wolf, Food Sci. Technol., 2011, 44, 1207-1211. 
24 J. M. Rodriguez Patino and A. M. R. Pilosof, Food Hydrocolloids, 2011, 25, 1925-1937.

25 H. Bunjes, K. Westesen and M. H. J. Koch, Int. J. Pharm., 1996, 129, 159-173.

26 M. Knoester, P. De Bruijne and M. Van den Tempel, Chem. Phys. Lipids, 1972, 9, 309-319.
27 B. Siekmann and K. Westesen, Colloids Surf., B, 1993, 3, 159-175.

28 I. T. Norton, C. D. Lee-Tuffnell, S. Ablett and S. M. Bociek, J. Am. Oil Chem. Soc., 1985, 62, 1237-1244.

29 D. Johansson and B. Bergenstahl, J. Am. Oil Chem. Soc., 1995, 72, 205-215. 